\title{
Parameter Modeling Analysis and Experimental Verification on Magnetic Shielding Cylinder of All-Optical Atomic Spin Magnetometer
}

\author{
Hong Zhang, ${ }^{1,2}$ Sheng Zou, ${ }^{1,2}$ Xi-Yuan Chen, ${ }^{1,2}$ and Wei Quan ${ }^{3}$ \\ ${ }^{1}$ School of Instrument Science and Engineering, Southeast University, Nanjing 210096, China \\ ${ }^{2}$ Key Laboratory of Micro-Inertial Instrument and Advanced Navigation Technology of Ministry of Education, \\ Southeast University, Nanjing 210096, China \\ ${ }^{3}$ Science and Technology on Inertial Laboratory, Beihang University, Beijing 100191, China \\ Correspondence should be addressed to Xi-Yuan Chen; chxiyuan@seu.edu.cn
}

Received 2 January 2015; Revised 2 April 2015; Accepted 8 April 2015

Academic Editor: Jesus Corres

Copyright (C) 2015 Hong Zhang et al. This is an open access article distributed under the Creative Commons Attribution License, which permits unrestricted use, distribution, and reproduction in any medium, provided the original work is properly cited.

\begin{abstract}
The ultrahigh sensitivity atomic spin magnetometer as the magnetic measurement sensor has received much concern. The performance of the magnetic shielding cylinder is one of the key factors constraining the atomic spin magnetometer's sensitivity. In order to effectively improve the performances of the magnetic shielding, the parameter optimization models of the magnetic shielding cylinder were established in this paper. Under the condition of changing only one parameter while the others keeping constant, the effects of various parameters influencing the axial shielding coefficient were comprehensively analyzed, and the results showed that the smaller the innermost length, the innermost radius, and the radial spacing were, and the greater the axial spacing was, the better the shielding performance could be obtained. According to these results and the actual needs, the magnetic shielding cylinder was optimally designed, and then the shielding effects were simulated via the software Ansoft. The simulation results showed that the optimized magnetic shielding cylinder had the advantages of small size, high shielding performance, and lager uniformity than that of the nonoptimized one. The actual measurement results showed that the residual magnetism in the optimized magnetic shielding cylinder was below $0.1 \mathrm{nT}$, which was $2 \sim 4$ times lower than the nonoptimized one.
\end{abstract}

\section{Introduction}

The ultrasensitive magnetometers are widely used in many disciplines, such as basic physics [1-4], biology [5, 6], medical imaging $[7,8]$, and material science and geography $[9,10]$. Recently, with the intensive study of the atomic spin theory and the rapid developments of quantum control technology and photoelectric detection technology, the ultrahigh sensitivity spin-exchange relaxation free (SERF) [11-13] atomic magnetometer emerges as the times require. Essentially, the SERF atomic spin magnetometer is a magnetic field measurement device, which is based on the hyperfine levels atomic transition and works at the environment with a weak magnetic field [14]. It is extremely important for the SERF atomic spin magnetometer to isolate the interferences from the external magnetic field, when it is implementing the ultrahigh sensitive magnetic measurement $[15,16]$. Therefore, it puts forward strict demands to the performances of the magnetic shielding. Generally, the magnetic shielding is made from ferromagnetic material with high permeability $[17,18]$, such as $\mu$-metal, and the shapes are mainly spherical, cylindrical, rectangular, and so forth. A closed cylindrical shell of finite length magnetic shielding is used in the SERF atomic spin magnetometer to insulate the magnetic field interference. The existing literatures about the magnetic shielding design mainly analyze the apertures $[19,20]$, the relative permeability, the shape, and the thickness of the shielding impacting on the shielding performance [21, 22]. However, few researches have been done on the parameters optimization of the magnetic shielding parameter, such as 


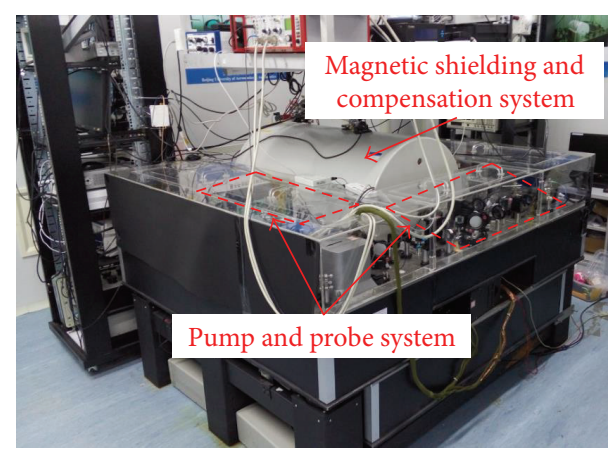

FIgURE 1: The SERF atomic spin magnetometer device.

length, radius, radial, and axial clearance. Currently, the designs of the magnetic shielding basically depend on experience, and there is lack of a complete optimization model.

Aiming at the problem of the atomic spin magnetometer's sensitivity being restricted by the magnetic shielding cylinder, the parameter optimization method and models for the magnetic shielding cylinder were proposed and established, respectively, under the condition of comprehensively considering the various factors affecting the shielding performance. Through the finite element analysis software, the shielding effects of the optimized and the nonoptimized magnetic shielding cylinder were simulated, respectively, and the optimization model and the simulation results were verified by experiments.

\section{Parameter Optimization Models of Multilayered Magnetic Shielding Cylinder}

2.1. Composition and Principle of SERF Atomic Spin Magnetometer. The SERF atomic spin magnetometer is mainly composed by the pump and probe system, the magnetic shielding and magnetic compensation system, the alkalimetal cell, and the heating system. The SERF atomic spin magnetometer device is shown in Figure 1.

The magnetic shielding is used to isolate the external magnetic field. The cell, which is heated to $180^{\circ} \mathrm{C}$ by the heating system, contains a droplet of alkali-metal atoms, several atm of ${ }^{4} \mathrm{He}$ and several torr $\mathrm{N}_{2}$. A circularly polarized pump beam, which is turned to the center of the D1 line of the alkali-metal atoms, is perpendicular to the linearly polarized probe beam. The output signal is modulated and demodulated by the photoelastic modulation (PEM) and the lock-in amplifier, respectively.

Generally, the relaxation time includes the longitudinal relaxation time $T_{1}$ and the transverse relaxation time $T_{2}$ :

$$
\begin{aligned}
& \frac{1}{T_{1}}=\frac{R_{\mathrm{tot}}}{Q\left(P^{e}\right)}+R_{\mathrm{wall}}, \\
& \frac{1}{T_{2}}=\frac{1}{T_{1}}+\frac{1}{q_{\mathrm{se}}} R_{\mathrm{se}}+R_{\mathrm{gr}} .
\end{aligned}
$$

Here $R_{\text {tot }}$ is the total relaxation time, $Q\left(P^{e}\right)$ is the slowing factor, $R_{\text {wall }}$ is the wall-collision relaxation rate, $1 / q_{\mathrm{se}}$ is

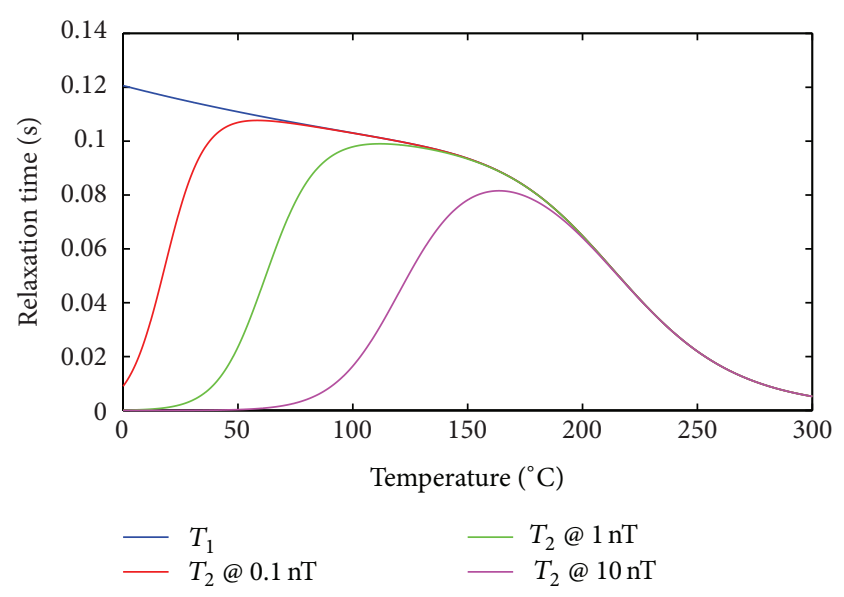

FIGURE 2: The temperatures realizing the SERF regime, under the different magnetic field environments.

the spin-exchange broadening factor, $R_{\mathrm{se}}$ is the spin-exchange relaxation rate, and $R_{\mathrm{gr}}$ is the broadening.

When the electrons stay in the SERF regime, one of the most important characteristics is that the spin exchange is suppressed; at this moment, $T_{2}$ is approximately equal to $T_{1}$. Under the different magnetic field environments, the temperatures realizing the SERF regime are shown in Figure 2.

Figure 2 shows that with increasing magnetic field, it needs a higher temperature to achieve the SERF regime. For example, at a $0.1 \mathrm{nT}$ and a $1 \mathrm{nT}$ magnetic field, the temperatures of realizing the SERF regime are approximately $120^{\circ} \mathrm{C}$ and $180^{\circ} \mathrm{C}$, respectively, while, in the case of a $10 \mathrm{nT}$ magnetic field existing, the temperature realizing the SERF regime is about $230^{\circ} \mathrm{C}$. For a $50000 \mathrm{nT}$ geomagnetic field, if there is no magnetic shielding, the SERF atomic spin magnetometer cannot work due to the extremely high temperature required. Thus, using the magnetic shielding to decrease the geomagnetic field is very important.

Optimizing the parameters of the magnetic shielding to obtain a high-performance and little volume magnetic shielding is vitally necessary.

2.2. Parameter Optimization Models. The shielding performance of the magnetic shielding cylinder is usually evaluated by shielding coefficient. The axial shielding coefficient becomes the key to restrict the shielding performance due to the axial shielding coefficient far less than the transverse shielding coefficient. Based on establishing the parameter optimization models, the influences of various parameters on the axial shielding coefficient were emphatically analyzed.

The transverse and the axial shielding coefficient formulas of $n$-layer magnetic shielding cylinder were described as follows:

$$
\begin{aligned}
& S_{\text {Ttot }}=S_{T n} \prod_{i=1}^{n-1} S_{T i}\left[1-\left(\frac{D_{i}}{D_{i+1}}\right)^{2}\right], \\
& S_{\text {Atot }}=S_{T n} \prod_{i=1}^{n-1} S_{T i}\left[1-\left(\frac{L_{i}}{L_{i+1}}\right)\right],
\end{aligned}
$$




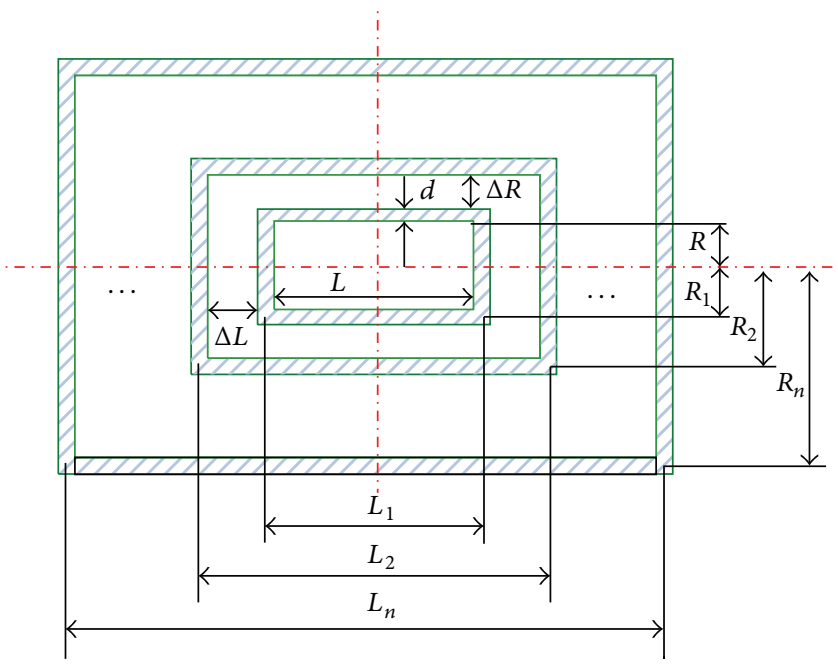

FIGURE 3: The axial profile map of the $n$-layer closed magnetic shielding cylinder.

where $S_{\text {Ttot }}$ is the total transverse shielding coefficient, $S_{T i}=$ $\mu_{r} d / D_{i}$ and $D_{i}$ are the transverse shielding coefficient and the average diameter at the $i$ th layer, respectively. $S_{\text {Atot }}$ is the total axial shielding coefficient, and $L_{i}$ is the average length at the $i$ th layer. The axial profile map of the $n$-layer closed magnetic shielding cylinder is shown in Figure 3.
From Figure 3 we know that the thickness $d$, the innermost length $L$, the innermost radius $R$, the radial interval $\Delta R$, and the axial clearance $\Delta L$ are contained between the neighboring shielding cylinder layers. Under the condition of considering these factors, (2) are improved and perfected, and the optimization models can be obtained:

$$
\begin{aligned}
& S_{\text {Ttot }}=\left(\frac{\mu_{r} d}{2}\right)^{n} \frac{\prod_{i=2}^{n}\left\{1-[(R+((2 i-3) / 2) d+(i-2) \Delta R) /(R+((2 i-1) / 2) d+(i-1) \Delta R)]^{2}\right\}}{\prod_{j=1}^{n}[R+(2 j-1 / 2) d+(j-1) \Delta R]}, \\
& S_{\text {Atot }}=\left(\frac{\mu_{r} d}{2}\right)^{n} \frac{\prod_{i=2}^{n}\{1-[(L+(2 i-3) d+2(i-2) \Delta L) /(L+(2 i-1) d+2(i-1) \Delta L)]\}}{\prod_{j=1}^{n}[R+((2 j-1) / 2) d+(j-1) \Delta R]} .
\end{aligned}
$$

Here $\mu_{r}$ is the relative permeability. According to (4), with other conditions steadfast, but changing only one parameter, the simulations of the influences from the various parameters on the axial shielding coefficient are shown in Figure 4.

Figure 4(a) shows that with the increasing of the relative magnetic permeability and the thickness of the cylinder, the shielding performances become better and better; Figure 4(b) describes that the axial shielding coefficient varies in inverse proportion to $\Delta R$. When $\Delta L$ changes below $20 \mathrm{~cm}$, the axial shielding coefficient varies greatly. However, if $\Delta L$ exceeds this range, the axial shielding coefficient varies slowly. Figure 4(c) delineates that with the increasing of the length and the radius of the innermost cylinder, the shielding coefficients become smaller and smaller.

The shielding coefficient of the magnetic shielding cylinder may also be characterized by the demagnetization factor, which is directly determined by the space ratio of the magnetic shielding cylinder:

$$
S \approx \frac{\left(4 N S_{T}+1\right)}{(1+1 / 2 P)}
$$

$$
\begin{aligned}
N & =\left[\frac{1}{\left(p^{2}-1\right)}\right] \\
& \cdot\left\{\frac{p}{\left(p^{2}-1\right)^{1 / 2} \ln \left[p+\left(p^{2}-1\right)^{1 / 2}\right]}-1\right\},
\end{aligned}
$$

where $N$ is the demagnetization factor, and $p=L / D$ is the space ratio of the magnetic shielding cylinder. The relationship between the demagnetization factor and the shielding coefficient and the relationship between the space ratio and the demagnetization factor are shown in Figures 5(a) and $5(\mathrm{~b})$, respectively.

Figure 5(a) shows that with the shielding coefficient increasing, the demagnetizing factor increases. Figure 5(b) indicates that with the space ratio increasing, the demagnetizing factor reduces, and at $L / D=2$, the biggest demagnetizing factor can be realized; meanwhile, the best shielding effect will also be obtained. This conclusion makes the parameter optimization model become more complete. 


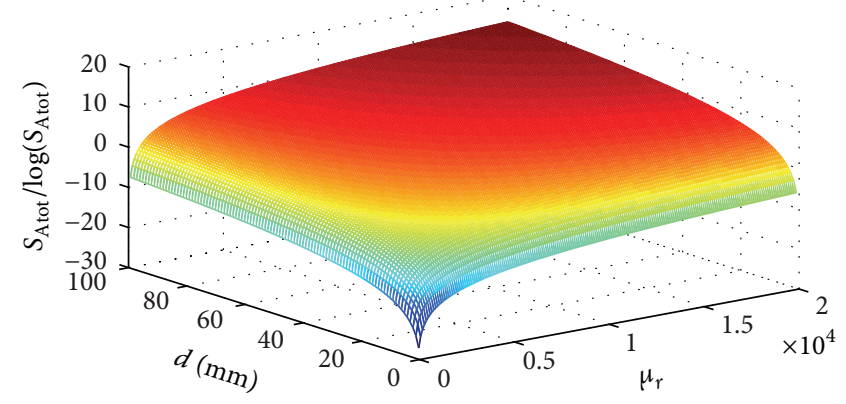

(a)

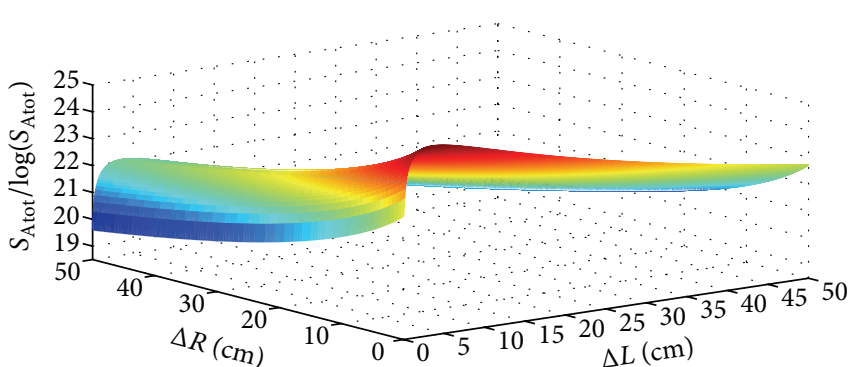

(b)

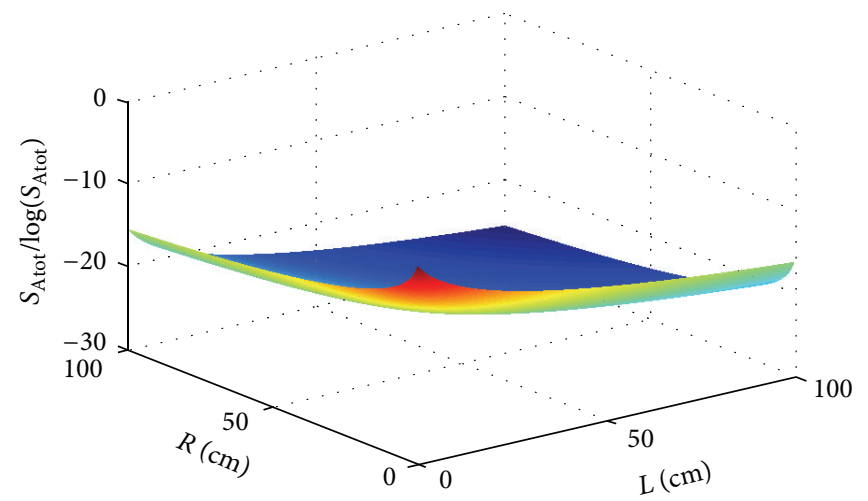

(c)

FiguRE 4: The simulations of the influences from the various parameters on the axial shielding coefficient. (a) The relationships among the thickness, the relative permeability, and the axial shielding coefficient; (b) the relationships among the radial interval, the axial interval, and the axial shielding coefficient; (c) the relationships among the radius of the inner cylinder, the length of the inner cylinder, and the axial shielding coefficient.

\section{Shielding Performance Simulation and Experimental Verification}

3.1. Materials Selection. The permeability performance of the magnetic material is expressed by the relative permeability. The higher the relative permeability is, the better the shield performance will be. Thus, the magnetic material with high relative permeability is used to fabricate the magnetic shielding. The commonly used magnetic materials and their relative permeability are listed in Table 1.

The Permalloy has been chosen to fabricate the magnetic shielding due to its highest relative permeability.

3.2. Simulation Modeling. The software Ansoft, which is based on the finite element method, is used to establish the simulation modeling. A pair of Helmholtz coils was applied to produce a uniform magnetic field about $23000 \mathrm{nT}$ along the axial direction. A five-layer closed magnetic shielding cylinder with trepanning was located in the center of the Helmholtz coils, as shown in Figure 6.

According to the foregoing analysis results and the actual requirements, the main design parameters of the magnetic shielding cylinder are listed in Table 2. The thickness of the magnetic shielding cylinder is $1 \mathrm{~mm}$, and the relative permeability is 10000 .
TABLE 1: The commonly used magnetic materials and their relative permeability.

\begin{tabular}{lc}
\hline Magnetic material & Relative permeability $\mu_{r}$ \\
\hline Cast iron & $200 \sim 400$ \\
Silicon steel sheet & $7000 \sim 10000$ \\
Ni-Zn ferrite & $10 \sim 1000$ \\
Mn-Zn ferrite & $300 \sim 50000$ \\
Permalloy & $2 \times 10^{4} \sim 2 \times 10^{5}$ \\
\hline
\end{tabular}

TABLE 2: The main design parameters of magnetic shielding.

\begin{tabular}{lcccc}
\hline Name & $R / \mathrm{mm}$ & $\Delta R / \mathrm{mm}$ & $L / \mathrm{mm}$ & $\Delta L / \mathrm{mm}$ \\
\hline Optimization & 120 & 11.5 & 480 & 250 \\
Nonoptimization & 210 & 10 & 626 & 100 \\
\hline
\end{tabular}

3.3. Analysis and Discussion. The CTM-6W single-axis fluxgate, which is developed by National Institute of Metrology of China, was used to measure the residual magnetism. The resolution and the measurement range of the fluxgate are $0.2 \mathrm{nT}$ and $0 \mathrm{nT} \sim 99999.9 \mathrm{nT}$, respectively. The test points were set along the axial direction with $1 \mathrm{~cm}$ intervals. The residual magnetism was measured by the probe positive and reverse placed, respectively. The two measurement results 


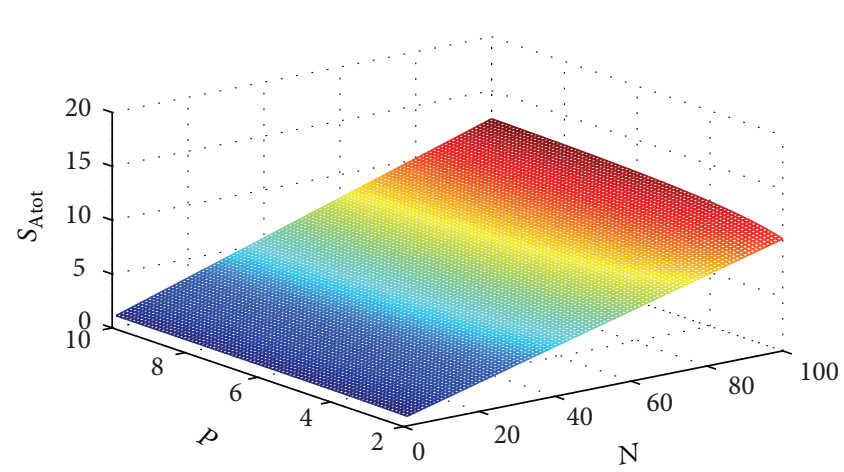

(a)

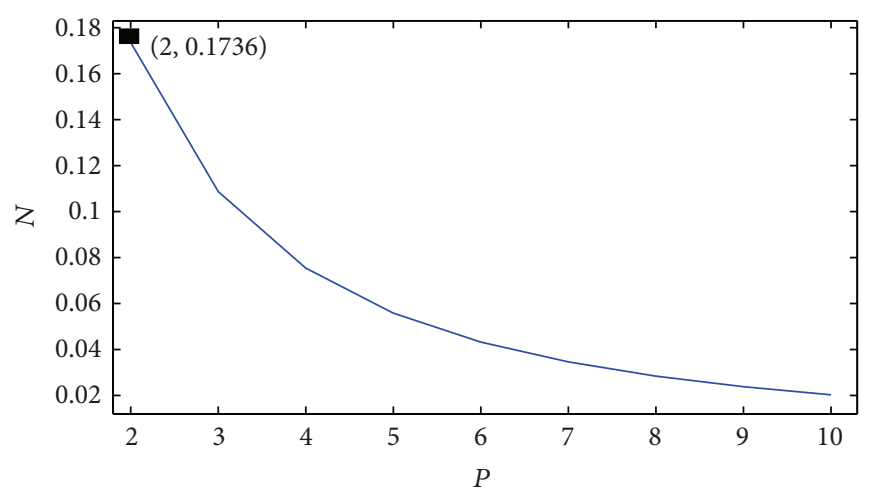

(b)

FIGURE 5: The space ratio of the magnetic shielding cylinder influencing the shielding performance. (a) The relationship between the demagnetization factor and the shielding coefficient. (b) The relationship between the space ratio and the demagnetization factor.

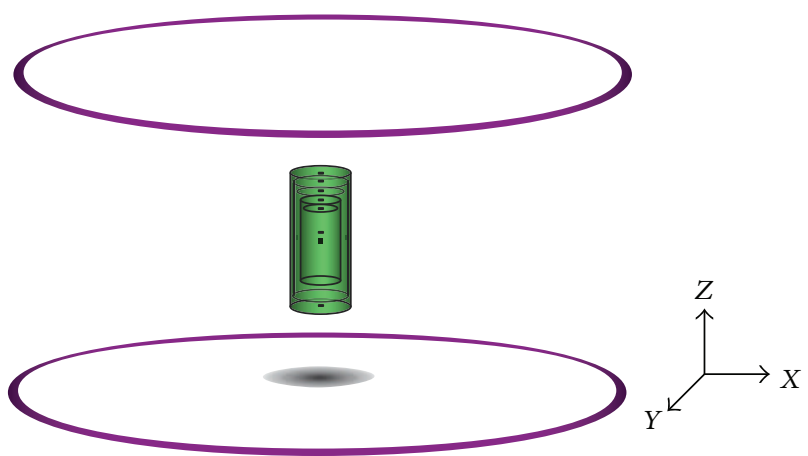

(a)

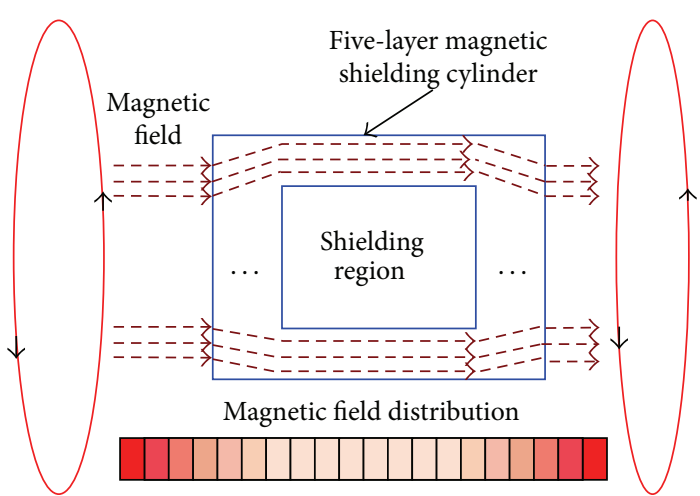

(b)

FIGURE 6: The simulation model of the magnetic shielding cylinder. (a) The simulation models. (b) Sectional view of the simulation model.

were subtracted in order to eliminate the offset value of the fluxgate, and the average value was the final result. The material objects of the nonoptimization magnetic shielding, which is used in the first generation SERF atomic spin magnetometer, and the optimization one, which will be used in the second generation magnetometer, are shown in Figures 7 (a) and 7(b), respectively. The simulation results and the test results of the optimization design and the nonoptimization design are shown in Figure 8.

The simulation results show that the residual magnetic field in the optimized magnetic shielding cylinder is about $0.01 \mathrm{nT}$; however, the residual magnetic field in the nonoptimization magnetic shielding cylinder is about $0.23 \mathrm{nT}$. The experimental results showed that the residual magnetic field in the optimized magnetic shielding cylinder was less than $0.1 \mathrm{nT}$ and the uniformity of the magnetic field was very good; however, the residual magnetic field in the nonoptimization magnetic shielding cylinder was between $0.2 \sim 0.4 \mathrm{nT}$. The enlarged drawing showed that there were some deviations between the experimental results and the simulation results. These deviations were mainly caused by three reasons. Firstly, the performance of the magnetic shielding was related to the annealing process, and the nonuniform distribution of the temperature on the magnetic shielding could impact the performance. The volume of the nonoptimization magnetic shielding cylinder was larger, and its shielding performance was easily influenced by the nonuniform distribution of the heat treatment temperature. Secondly, Permalloy was sensitive to the mechanical stress. In the processes of the machining, the transportation, and the assembling, it could not avoid the stress effects on the properties of the magnetic shielding. Thirdly, there were some reading errors during the operation. But the simulation results agreed with the experimental results without considering the influences of errors.

\section{Conclusion}

The various factors affecting the shielding performance were analyzed in this paper. Firstly, the parameter optimization formulas of the magnetic shielding cylinder were deduced, and the simulation results indicated that $\mu_{r}, d, \Delta L, L, R$ had a larger effect on the axial total shielding coefficient, while, compared with the other parameters, $\Delta R$ effected smaller. The magnetic shielding cylinder with small size had the 


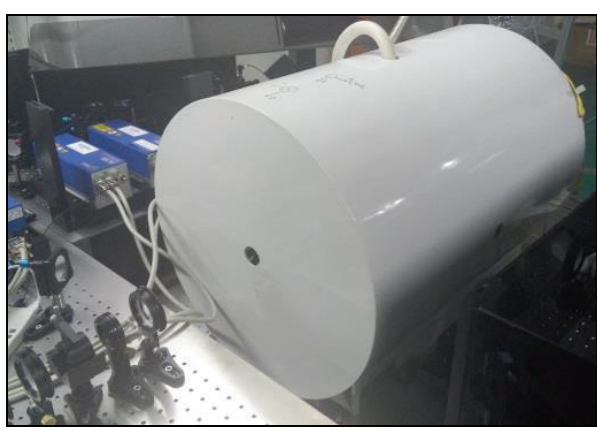

(a) The nonoptimization magnetic shielding

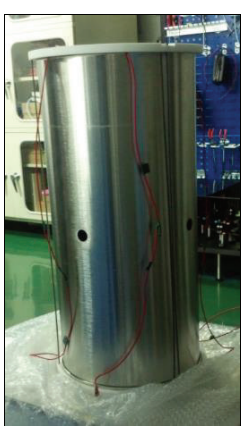

(b) The optimization magnetic shielding

FIgURE 7: The material objects of the nonoptimization magnetic shielding and the optimization one.

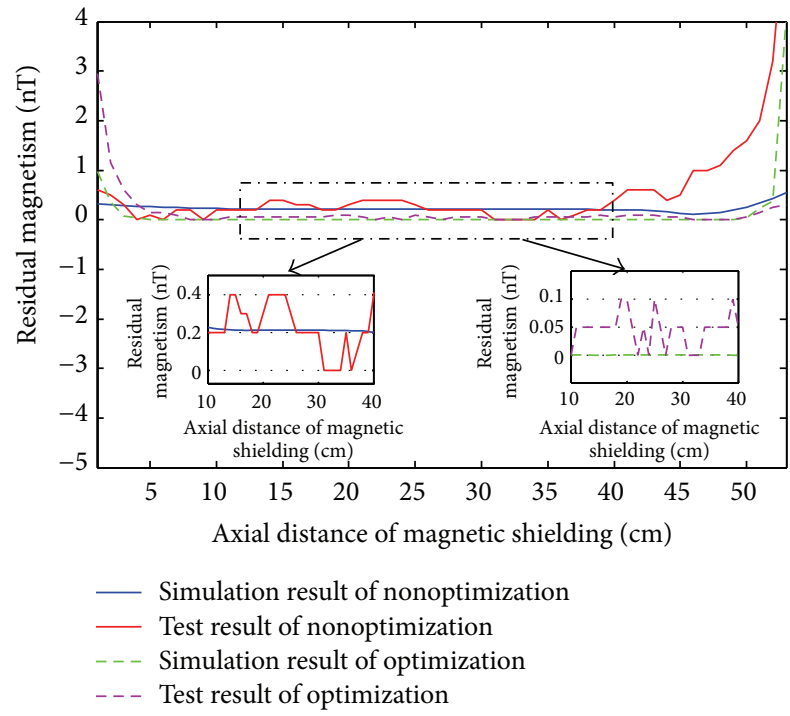

Figure 8: The simulation results and the test results of the optimization design and the nonoptimization design.

advantages of larger shielding coefficient and better shielding effect. Secondly, at $L / D=2$, the biggest demagnetizing factor could be realized. The correctness of the optimization model could be validated by simulations and experiments, and the optimized magnetic shielding cylinder was below $0.1 \mathrm{nT}$. The magnetic shielding cylinder designed by the optimization modeling approach described in this paper had the advantages of good shielding performance, small volume, light weight, and low cost, and it could provide theoretical guidance to the optimization design of the magnetic shielding structure.

\section{Conflict of Interests}

The authors declare that there is no conflict of interests regarding the publication of this paper.

\section{Acknowledgments}

This study was supported by the Major Program of the National Natural Science Foundation of China (Grant no. 61227902), the National Natural Science Foundation of China (Grant no. 51375087), and the Fundamental Research Funds for the Central Universities of Ministry of Education of China (Grant no. CXLX13_083).

\section{References}

[1] J. M. Brown, A New Limit on Lorentz- and CPT-Violating Neutron Spin Interactions Using a $\mathrm{K}^{3} \mathrm{He}$ Comagnetometer, Princeton University, Princeton, NJ, USA, 2011.

[2] L. Moi and S. Cartaleva, "Sensitive magnetometers based on dark states," Europhysics News, vol. 43, no. 6, pp. 24-27, 2012.

[3] D. Ren, L. Wu, M. Yan, M. Cui, Z. You, and M. Hu, "Design and analyses of a MEMS based resonant magnetometer," Sensors, vol. 9, no. 9, pp. 6951-6966, 2009.

[4] C. C. Lu, J. Huang, P. K. Chiu, S. L. Chiu, and J. T. Jeng, "Highsensitivity low-noise miniature fluxgate magnetometers using a flip chip conceptual design," Sensors, vol. 14, no. 8, pp. 1381513829, 2014.

[5] I. Savukov and T. Karaulanov, "Anatomical MRI with an atomic magnetometer," Journal of Magnetic Resonance, vol. 231, pp. 3945, 2013.

[6] H. B. Dang, A. C. Maloof, and M. V. Romalis, "Ultrahigh sensitivity magnetic field and magnetization measurements with an atomic magnetometer," Applied Physics Letters, vol. 97, no. 15, Article ID 151110, 2010.

[7] T. H. Sander, J. Preusser, R. Mhaskar, J. Kitching, L. Trahms, and S. Knappe, "Magnetoencephalography with a chip-scale atomic magnetometer," Biomedical Optics Express, vol. 3, no. 5, pp. 981990, 2012.

[8] R. Wyllie, M. Kauer, G. S. Smetana, R. T. Wakai, and T. G. Walker, "Magnetocardiography with a modular spin-exchange relaxation-free atomic magnetometer array," Physics in Medicine and Biology, vol. 57, no. 9, pp. 2619-2632, 2012.

[9] J. C. Fang, T. Wang, W. Quan et al., "In situ magnetic compensation for potassium spin-exchange relaxation-free magnetometer considering probe beam pumping effect," Review of Scientific Instruments, vol. 85, no. 6, Article ID 063108, 2014. 
[10] D. Sheng, S. Li, N. Dural, and M. V. Romalis, "Subfemtotesla scalar atomic magnetometry using multipass cells," Physical Review Letters, vol. 110, no. 16, Article ID 160802, 2013.

[11] I. K. Kominis, T. W. Kornack, J. C. Allred, and M. V. Romalis, "A subfemtotesla multichannel atomic magnetometer," Nature, vol. 422, no. 6932, pp. 596-599, 2003.

[12] S. J. Seltzer, Developments in Alkali-Metal Atomic Magnetometry, Princeton University, Princeton, NJ, USA, 2008.

[13] J. Kitching, S. Knappe, and E. A. Donley, "Atomic sensors-a review," IEEE Sensors Journal, vol. 11, no. 9, pp. 1749-1758, 2011.

[14] S. Appelt, A. Ben-Amar Baranga, C. J. Erickson, M. V. Romalis, A. R. Young, and W. Happer, "Theory of spin-exchange optical pumping of ${ }^{3} \mathrm{He}$ and ${ }^{129} \mathrm{Xe}$," Physical Review A, vol. 58, no. 2, pp. 1412-1439, 1998.

[15] T. W. Kornack, A Test of CPT and Lorentz Symmetry Using a $\mathrm{K}-3 \mathrm{He}$ Co-Magnetometer, Princeton University, Princeton, NJ, USA, 2005.

[16] R. K. Ghosh, Spin Exchange Optical Pumping of Neon and Its Applications, Princeton University, Princeton, NJ, USA, 2009.

[17] G. Planinšič, "Shielding of low-frequency magnetic interference in weak-field MRI by a single-layer cylindrical coil," Journal of Magnetic Resonance, vol. 126, no. 1, pp. 30-38, 1997.

[18] M. Duffy, S. Kulkarni, and S. Roy, "Improved shielding performance using high permeability electroplated thin films in printed circuit boards," IEEE Transactions on Magnetics, vol. 47, no. 10, pp. 4282-4285, 2011.

[19] L. Xuan, H. Dong, and C. Zhuo, "Compact multi-layer magnetic shields for chip-scale atomic devices," in Proceedings of the 8th Annual IEEE International Conference on Nano/Micro Engineered and Molecular Systems (IEEE NEMS '13), pp. 649652, Suzhou, China, April 2013.

[20] C.-Q. Jiao and S. Niu, "Shielding effectiveness of an apertured rectangular cavity against the near-field electromagnetic waves," Acta Physica Sinica, vol. 62, no. 11, Article ID 114102, 2013.

[21] T. J. Sumner, J. M. Pendlebury, and K. F. Smith, "Convectional magnetic shielding," Journal of Physics D: Applied Physics, vol. 20, no. 9, pp. 1095-1101, 1987.

[22] T. W. Kornack and M. V. Romalis, "Dynamics of two overlapping spin ensembles interacting by spin exchange," Physical Review Letters, vol. 89, no. 25, Article ID 253002, 2002. 

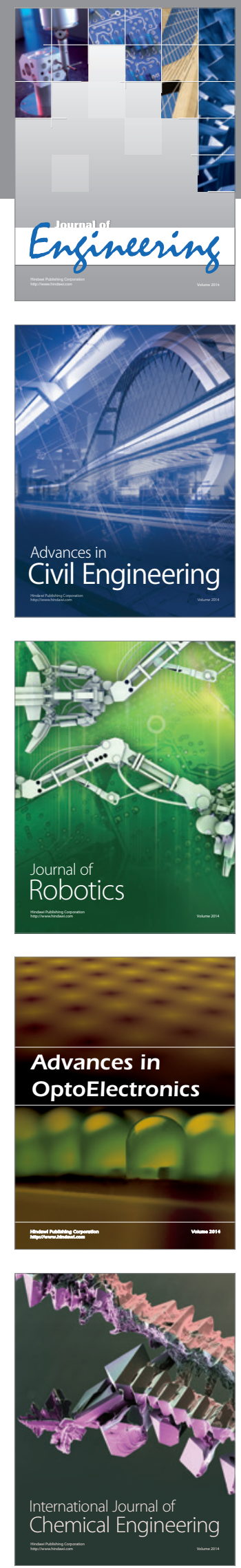

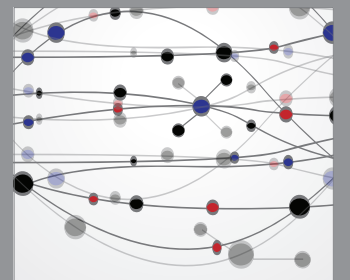

The Scientific World Journal
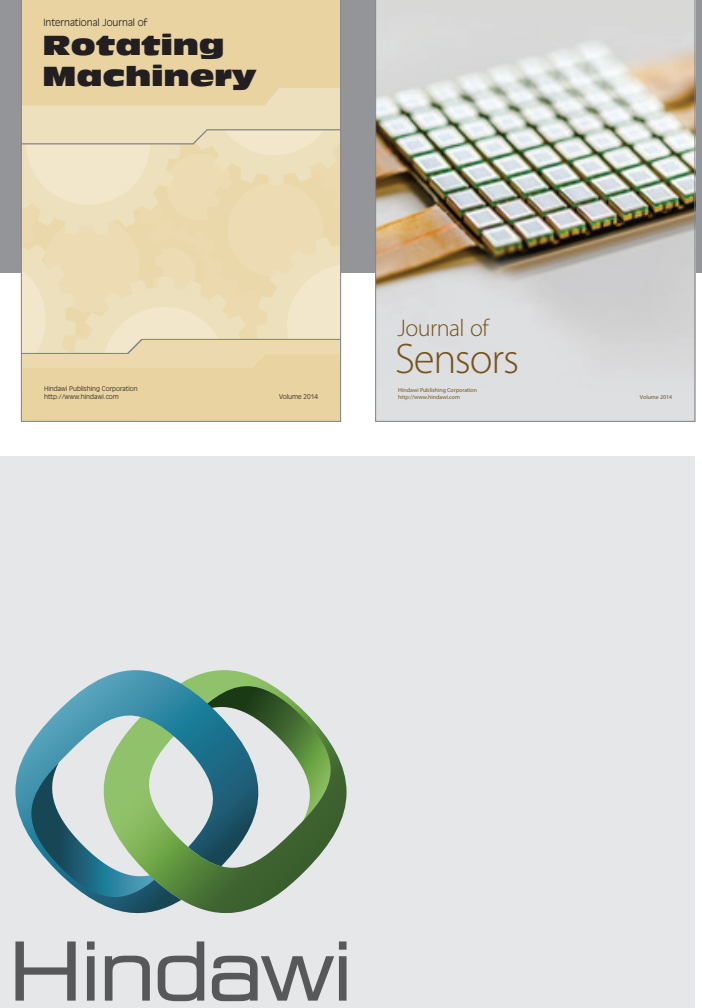

Submit your manuscripts at http://www.hindawi.com
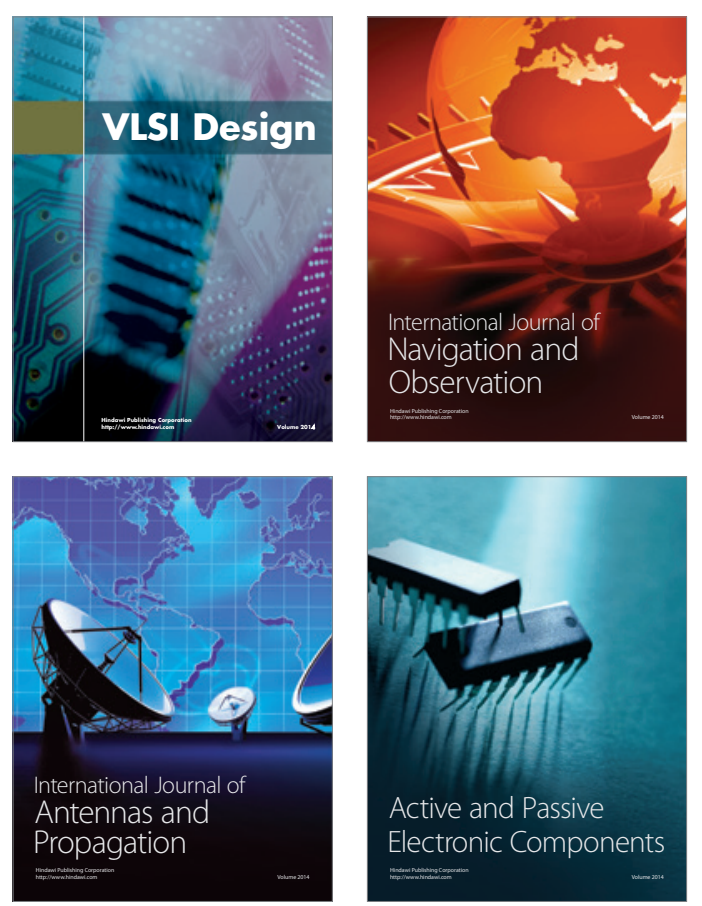
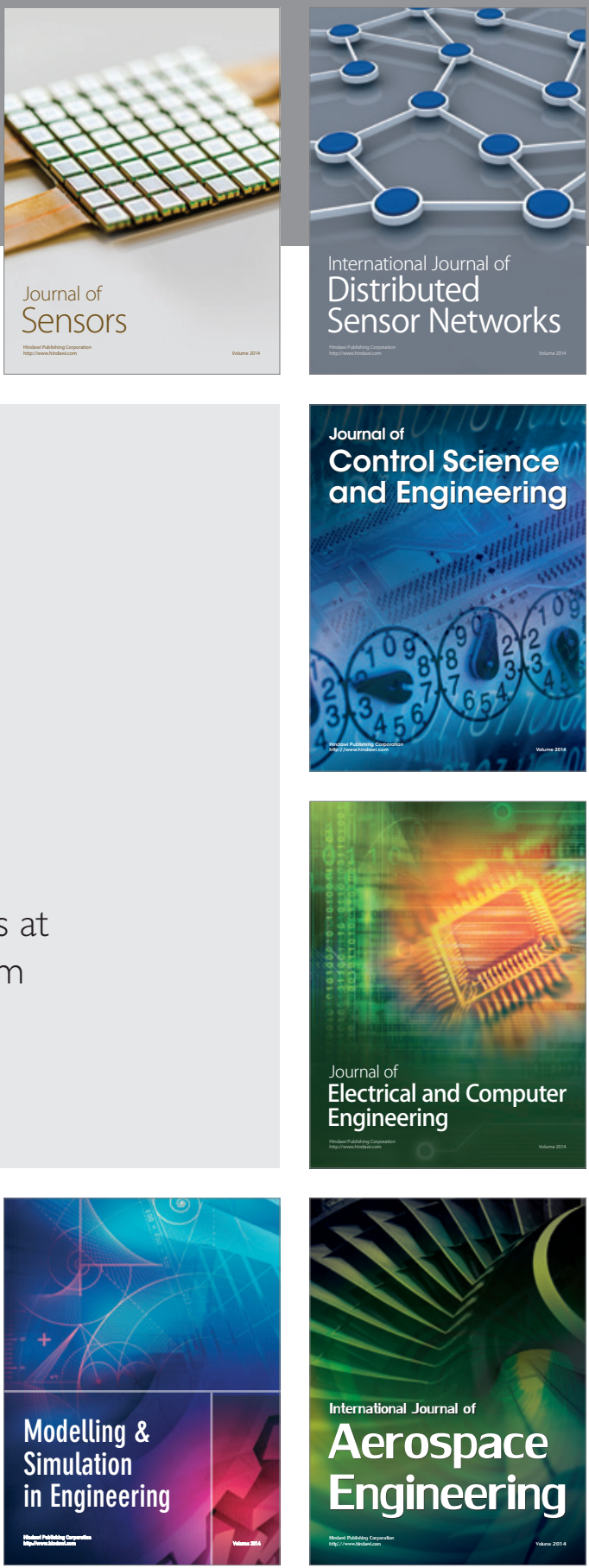

Journal of

Control Science

and Engineering
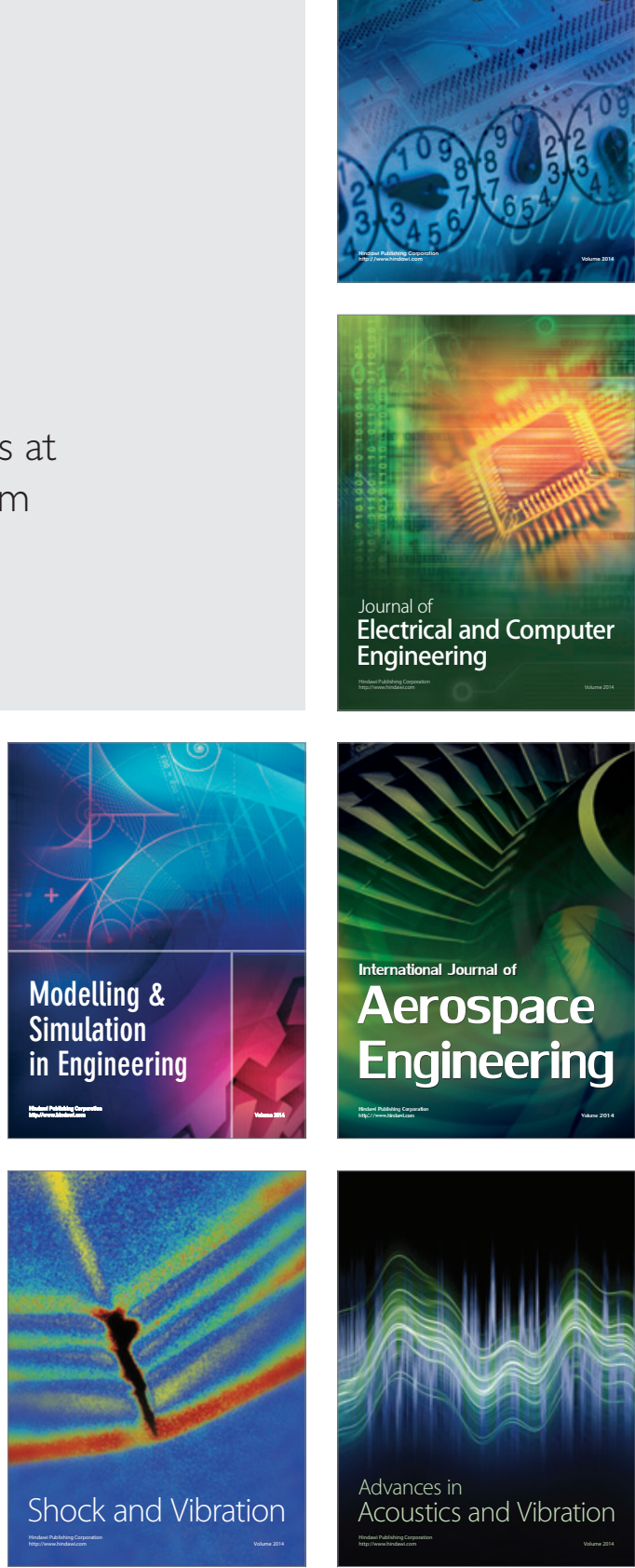